\title{
Portable magnetic tweezers device enables visualization of the three-dimensional microscale deformation of soft biological materials
}

\author{
Yali Yang ${ }^{1, *}$, Jun Lin ${ }^{2, *}$, Ryan Meschewski ${ }^{1}$, Erin Watson ${ }^{3}$, and Megan T. Valentine ${ }^{1}$ \\ ${ }^{1}$ Department of Mechanical Engineering, University of California-Santa Barbara, Santa Barbara, CA, USA, \\ ${ }^{2}$ Biomolecular Science and Engineering Program, University of California-Santa Barbara, Santa Barbara, $C A$, \\ USA, and ${ }^{3}$ NSF-NNIN Internship Program, University of California-Santa Barbara, Santa Barbara, CA, USA
}

BioTechniques 50:29-34 (July 2011) doi 10.2144/000113701

Keywords: magnetic tweezers; confocal microscopy; hydrogel; traction force; cytoskeleton; structure-mechanics relationships

${ }^{*}$ Y.Y. and J.L. contributed equally to this work.

We have designed and built a magnetic tweezers device that enables the application of calibrated stresses to soft materials while simultaneously measuring their microscale deformation using confocal microscopy. Unlike previous magnetic tweezers designs, our device is entirely portable, allowing easy use on microscopes in core imaging facilities or in collaborators' laboratories. The imaging capabilities of the microscope are unimpaired, enabling the 3-D structures of fluorescently labeled materials to be precisely determined under applied load. With this device, we can apply a large range of forces $(\sim 1-1200 \mathrm{pN})$ over micron-scale contact areas to beads that are either embedded within 3-D matrices or attached to the surface of thin slab gels. To demonstrate the usefulness of this instrument, we have studied two important and biologically relevant materials: polyacrylamide-based hydrogel films typical of those used in cell traction force microscopy, and reconstituted networks of microtubules, essential cytoskeletal filaments.

Microscale mechanical testing of soft biomaterials often requires specialized (and often custom-built) equipment, such as optical traps and magnetic tweezers $(1,2)$. The complexity of the design and calibration of these instruments has typically limited their use to single investigator laboratories, and very few devices have been successfully incorporated into high-resolution 3-D imaging platforms $(3-5)$. This has severely limited their use in determining structure-mechanics relationships in complex materials. To address this, we designed a portable magnetic tweezers device that enables the application of controlled forces to soft materials while they are simultaneously imaged using a commercial laser scanning confocal microscope. Our design does not require permanent microscope modifications nor specialized data acquisition hardware, thus facilitating its use in core facilities and collaborators' laboratories. This instrument can be built at low cost and operated without specialized optics expertise, enabling its broad use in a variety of interdisciplinary biomedical research and teaching settings.

\section{Materials and methods}

Portable magnetic tweezers design In developing this device, the following design requirements were enforced: (i) The device must be lightweight, inexpensive, and easy to assemble, calibrate, and move. (ii) The instrument must be mechanically stable and capable of generating reproducible, precise forces in the imaged sample volume. (iii) To achieve the strongest magnetic field within the visualized field of view, the magnets must be placed as close as possible to the sample, without interfering with the normal operation of the microscope. To achieve these goals, we designed a magnetic tweezers device consisting of two neodymium iron boron rare earth magnets $\left(\mathrm{N} 45,0.25 \times 0.25 \times 1 \mathrm{in}^{3}\right.$; Applied Magnets, Plano, TX, USA) mounted onto a two-axis translation stage, allowing the distance between the magnetic pole pieces and sample to be controllably varied (Figure 1).
To improve the portability of our design, we employed lightweight manual translation stages to position the magnets with respect to the sample; however, the manual stages could be replaced with motorized linear stages using standalone DC servo motors if faster force switching is required.

A modified sample holder plate was machined to elevate the sample and minimize the distance between the magnets and imaged field of view. Prior to loading the sample, the magnets were positioned directly over the objective lens and centered with respect to the optical axis. The magnets were then retracted to allow the slide to be loaded, and the focus adjusted until the edge of the sample chamber could be visualized at the edge of the field of view. The height of the device was adjusted until the magnets were centered vertically about the center of the slide. The magnets were then moved toward the slide until they were just touching the slide edge; this position was recorded as $[x, y]=[0,0]$. In our sideways pulling design (Figure 1A), the magnets are typically placed within several millimeters of 


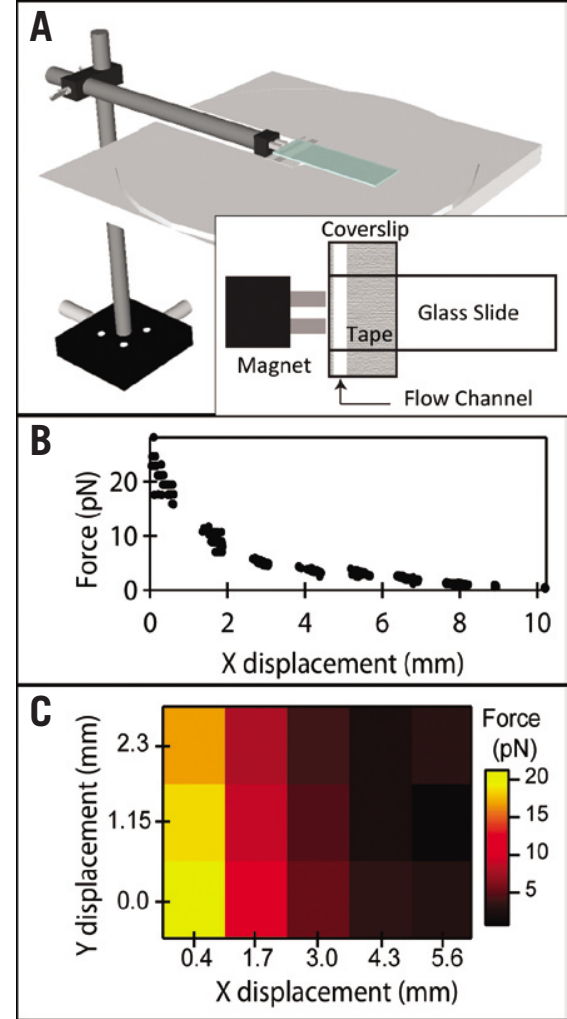

Figure 1. Design and calibration of the portable magnetic tweezers device. A pair of neodymium iron boron rare earth magnets are mounted on a two-axis translation stage to allow for precise positioning with respect to the imaged field of view. Inset: The magnets are placed in contact with the slide, thereby maximizing the magnetic force at the imaged field of view. A thin $(\sim 1-\mathrm{mm})$ tape strip is used to form the left-hand edge of the flow channel. (B) Applied force as a function of $x$-displacement along the $y=0$ line for the $d=$ $4.5-\mu \mathrm{m}$ beads. The force peaks as $x \rightarrow 0$, and decreases monotonically with a $\sim 75 \%$ decrease at a separation distance of $\sim 3 \mathrm{~mm}$. (C) A heat map showing the 2-D mapping of applied force for the $d=4.5-\mu \mathrm{m}$ beads; because the magnetic force field is symmetric around the $y=0$ line, only the positive $y$ values are shown.

the sample when using $20 \times$ air $($ N.A. $=0.7)$ or $60 \times$ oil-immersion (N.A. $=1.4)$ lenses (UPlanApo, Olympus, Center Valley, PA, USA). The shape of some high-magnification lenses may increase this distance, thereby diminishing the maximum force that can be achieved; however, custom pole pieces could be manufactured to enhance and focus the magnetic fields if both high magnification and high force are required (6).

In contrast to our design, previous magnetic tweezers devices have typically required microscope modifications and additional instrumentation to manipulate the magnetic fields and apply force. Several designs employ custom-machined single- or multipole electromagnets, electronics to controllably vary coil current and phase, and cooling systems to remove excess heat (7-10). When implemented with feedback control, these devices can manipulate small magnetic beads in three dimensions; however, the systems are too cumbersome to be easily portable. Many permanent magnet-based devices, which avoid the need for high-current electronics and cooling, employ a vertical pulling geometry that allows the magnets to nearly touch the sample, thus maximizing the pulling force $(1,11,12)$. However, this requires significant microscope modification and can limit the imaging capabilities of the instrument, since the condenser optics must be removed to allow the magnets to be placed directly above the sample. To our knowledge, none of these devices has been used in a portable format (11-13).

Preparation of hydrogel samples Coverslips were prepared as previously described, with minor modifications (14). Coverslips $\left(22 \times 40 \mathrm{~mm}^{2}\right.$, \#1.5 thickness; Cat. no 2940-224; Corning, Corning, NY, USA) were cleaned with $70 \%$ ethanol in water, and soaked in 2\% 3-aminopropyltrimethoxysilane (Cat. no. AC31325-1000; Acros Organics, Fair Lawn, NJ, USA) in isopropanol for $10 \mathrm{~min}$ under constant stirring. Coverslips were washed extensively in distilled deionized water $\left(\mathrm{ddH}_{2} 0\right)$ and oven-dried. Once cooled, coverslips were submerged in a $1 \%$ glutaraldheyde solution (Cat. no. BP2547-1; Fisher Scientific, Pittsburgh, PA, USA) for 30 min under constant stirring. Activated coverslips were washed extensively in $\mathrm{ddH}_{2} \mathrm{O}$, dried, and then stored in a dust-free container for $\leq 3$ weeks.

Hydrogel substrates were prepared as previously described, with minor modifications to allow covalent attachment of biotin moieties at the gel surface (14). Briefly, stock solutions of $40 \%$ acrylamide and $2 \%$ bis-acrylamide were diluted to obtain gels of desired stiffness (15). Diluted solutions were degassed under sonication for $20 \mathrm{~min}$, and 100-n m diameter yellowgreen fluorescent particles were added to a final concentration of $0.05 \%$. Biotin-conjugated BSA (Vector Labs, Burlingame, CA, USA) was covalently coupled to succinimidyl-esters of Acryloyl-X (Invitrogen, Carlsbad, CA, USA). Excess reactants were removed by spin filtration, and the purified protein added to the acrylamide solution at a final concentration of $\sim 0.5 \mathrm{mg} / \mathrm{mL}$ (16). To initiate polymerization, ammonium persulfate (Cat. no. 161-0700; Bio-Rad, Hercules, CA, USA) and TEMED (Cat. no. 161-0800; Bio-Rad) were added to final concentrations of $0.05 \%$ and $0.15 \%$ respectively.
Ten microliters of the activated acrylamide solution was applied to the center of a $25.4 \times 76.3 \mathrm{~mm}^{2}$ hydrophobically treated microscope glass slide. A glutaraldehyde-activated glass coverslip was placed on top of the droplet in a crosswise fashion to create a nominal gel area of $\sim 22$ $\times 25 \mathrm{~mm}^{2}$. Gentle pressure was applied to the coverslip to evenly spread the gel and remove air bubbles; the resulting hydrogel was $\sim 15-20 \mu \mathrm{m}$ thick. The gel was left undisturbed for $\geq 10 \mathrm{~min}$ at room temperature to allow polymerization to occur, then the coverslip with attached gel was gently removed from the slide using a clean razor blade.

A $200-\mu \mathrm{L}$ droplet of $45-\mu \mathrm{m}$ streptavidin-coated magnetic beads (Spherotech, Lake Forest, IL, USA) at $0.125 \% \mathrm{w} / \mathrm{v}$ was placed on one edge of a freshly prepared biotin-BSA-conjugated PAA hydrogel. The remaining gel surface was hydrated with $\sim 300 \mu \mathrm{L} \mathrm{ddH_{2 }} \mathrm{O}$. The coverslip was incubated for $5 \mathrm{~min}$ at room temperature to induce strong binding of the magnetic beads to the gel surface. Each coverslip was then gently washed with $\mathrm{ddH}_{2} \mathrm{O}$ to remove excess beads. Gel-coated coverslips were mounted onto a clean glass slide using double-sided tape (thickness 300 $\mu \mathrm{m})$, hydrated with water, sealed with wax, and used immediately.

Preparation of microtubule samples Entangled microtubule networks were generated by combining the following reagents on ice: unlabeled tubulin, rhodamine-labeled tubulin, $1 \mathrm{mM}$ GTP, $1 \mathrm{mM}$ DTT, $10 \%$ (v/v) DMSO, taxol and PEM80 (80 mM PIPES, $4 \mathrm{mM} \mathrm{MgCl}$, 1 mM EGTA; pH 6.9). The molar ratios of rhodamine-labeled tubulin to total tubulin is $1: 6$, and taxol to total tubulin is 1:2.4.5- $u \mathrm{~m}$ tosyl-activated magnetic beads and $\sim 2.5-\mu \mathrm{m}$ latex beads are added to the ice-cold mixture at final concentrations of $\sim 10^{6}$ beads $/ \mathrm{mL}$ and $\sim 3 \times 10^{7}$ beads $/ \mathrm{mL}$, respectively. The solution is then loaded into small rectangular capillary tubes $(0.1 \times$ $1 \times 50 \mathrm{~mm}^{3}$; model no. BMS-010-1-090-50, Friedrich \& Dimmock, Inc., Millville, NJ, USA) and incubated at $35^{\circ} \mathrm{C}$ for $\sim 1 \mathrm{~h}$ under constant rotation to prevent the magnetic beads from settling onto one side of the capillary tube.

\section{Confocal imaging}

All confocal microscopy images were collected using an inverted Fluoview 500 laser scanning system (Olympus). For purposes of calibration, samples were visualized using brightfield illumination $(10 \times$ objective lens, N.A. $=0.4$, no digital zoom, $1243 \mathrm{~nm} /$ pixel magnification). For 

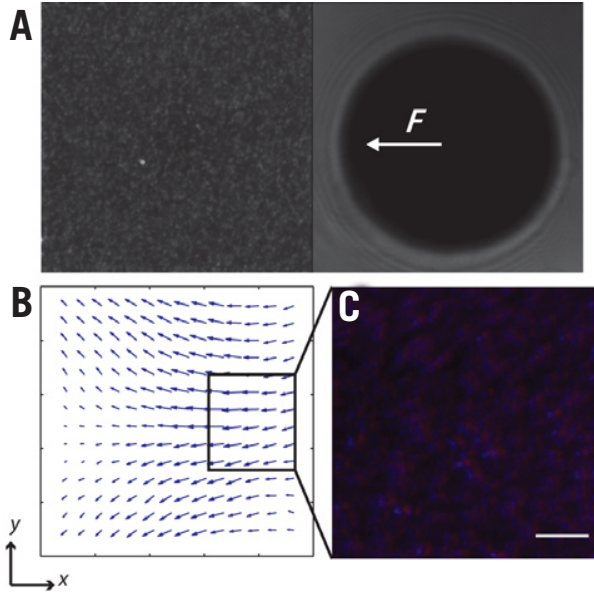

Figure 2. Deformation of soft hydrogel under stress. (A) Representative microscopy image for hydrogel characterization. Left panel shows confocal image of 100-nm fluorescent beads embedded in a polyacrylamide gel with $50-\mathrm{Pa}$ shear modulus, and subjected to a force of $\sim 1$ nN. Right panel shows brightfield image of 45$\mu \mathrm{m}$ magnetic bead, which is attached to the top surface of the gel using a biotin-streptavidin linkage. The white arrow indicates the direction of the applied force, F. (B) The deformation field of the gel in a $z$-plane $\sim 1 \mu \mathrm{m}$ below the bottom of the bead surface is determined by the crosscorrelation of images collected before and after the force is applied. (C) Two-color overlay of the force-on (blue) and force-off (red) images demonstrates gel displacement at the rear edge of the magnetic bead (area indicated by box in panel B). Scale bar $=5 \mu \mathrm{m}$.

each bead type, a time series of 2-D scans was collected using a scan size of 1024 $\times 1024$ pixels $^{2}$ and rate $=4$ scans $/ \mathrm{s}$. To visualize hydrogel deformation, magnetic and fluorescent beads ( $505 \mathrm{~nm}$ excitation; $515 \mathrm{~nm}$ emission) were directly visualized using 488-nm laser excitation and transmitted light $(20 \times$ objective lens, N.A. $=$ 0.7 , digital zoom $=10 \times$, magnification $62 \mathrm{~nm} /$ pixel). For hydrogel characterization, a 3-D image stack was collected for both the transmitted light and fluorescence channels using a scan size of 1024 $\times 1024$ pixels $^{2}$ at a rate of 3.26 s/scan. For cytoskeletal network characterization, rhodamine-labeled microtubules, magnetic beads and latex beads were visualized using 561-nm laser excitation and transmitted light $(60 \times$ oil-immersion objective lens, N.A. $=1.4$, digital zoom $=4 \times$, magnification $103.6 \mathrm{~nm} /$ pixel). For each gel, a time series of 2-D images was collected for both the transmitted light and fluorescence channels using a scan size of $512 \times$ 512 pixels $^{2}$ at a rate of $1.12 \mathrm{~s} / \mathrm{scan}$.

\section{Image processing}

For calibration, each image was converted to an 8-bit binary TIFF image after thresholding. For the 4.5- $\mu \mathrm{m}$ and $20.8-\mu \mathrm{m}$ beads, the ImageJ plugin SpotTracker 2D was used to determine the center of each bead in each frame. Under these conditions, the $45-\mu \mathrm{m}$ beads moved slightly during the scan, leading to a noncircular shape. For these images, the bead center was determined using the built-in ImageJ tracing tool to manually select bright features and the measure tool to determine the center position. In all cases, the instantaneous bead velocity was determined by dividing the distance traveled between two successive frames by the elapsed time. For hydrogel characterization, MATLABbased particle imaging velocimetry code (MPIV, developed by N. Mori and K.-A. Chang; www.oceanwave.jp/softwares/ mpiv), was used to identify the movement of embedded beads with sub-pixel accuracy, using the minimum quadratic differences (MQD) algorithm and a displacement grid spacing of 128 pixels. The resultant displacement vectors were filtered and interpolated using Kriging interpolation.

\section{Results and discussion}

Portable magnetic tweezers enables precision application of forces over large sample area

The force that can be exerted by this instrument depends not only on the shape and strength of the permanent magnets, but also on the size and iron content of the magnetic particles that are embedded in the sample. To establish the range of forces that can be applied with the portable device, three commercial particle types were used: tosyl-activated magnetic dynabeads with diameter $d=4.5 \mu \mathrm{m}$ (Invitrogen), and

Table 1. Summary of maximal force data.

\begin{tabular}{ccc|} 
Particle diameter $(\boldsymbol{\mu m})$ & Iron content (\%) & Maximum force $(\mathbf{p N})$ \\
\hline 4.5 & $20 \%$ & 25 \\
20.8 & $3 \%$ & 100 \\
45 & $4.9 \%$ & 1200 \\
\hline
\end{tabular}

By varying particle size and iron content, it is possible to vary the applied force over $\sim 3$ orders of magnitude. Here, the maximum applied forces for three commercial bead types are summarized. streptavidin-coated magnetic beads with $d$ $=20.8 \mu \mathrm{m}$ or $45 \mu \mathrm{m}$ (Spherotech). For each bead type, particles were added to a $70 \%$ glycerol solution in water to a final bead concentration of $0.01 \%$. Bead suspensions were introduced into a thin microscope chamber consisting of a clean, untreated glass coverslip and slide separated by layers of double-sided tape ( 200- $\mu$ m thickness) and sealed with ultra-high-vacuum grease. The magnets were moved into the $[0,0]$ position and the field of view adjacent to the proximal tape edge, where the magnetic field is largest, was visualized. The velocity for each bead type was measured and the force $F$ was determined using Stoke's Law: $F=3 \pi \eta d v$ (17). The maximal force increased monotonically with particle size, and varied from 25 to $1200 \mathrm{pN}$ (Table 1).

To determine the spatial variation in applied force, bead velocity is measured as a function of distance from the $[0,0]$ position at a fixed bead radius (Figure 1, B and C). The magnitude of applied force decreases monotonically, and although this decay is not purely exponential, there is a characteristic length scale on the order of several millimeters. For high-resolution imaging, this length scale is significantly larger than a typical field of view, facilitating nearly constant-force measurements within the imaging volume. At lower magnification, the force-displacement calibration curves can be used to enable multiple and simultaneous measurements at a range of forces within the same sample preparation.

Direct measurement of displacement fields in thin hydrogels

Hydrogels, or water-swollen polymer networks, are an important class of biomaterials whose structural and mechanical similarities to biological tissues have led to a number of medical applications (18-21) and have enabled their use as artificial extracellular matrix substrates for the culturing of adherent cells (22-25). By incorporating fluorescent markers into the gel substrates, it is possible to directly visualize cellinduced matrix deformation, and-if the mechanical properties of the substrates are known-to calculate the cell-induced forces. Polyacrylamide (PAA)-based hydrogels have been particularly useful in traction force measurements since the inexpensive gels exhibit stable mechanical properties, and have few nonspecific chemical interactions with proteins or cells. Moreover, it is possible to independently control their mechanical properties (by tuning polymer concentration and crosslinking), and interfacial chemistry (by coupling specific extracellular matrix proteins at the gel surface) (26). 


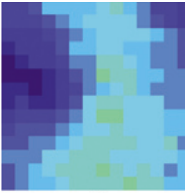

$z=-6 \mu m$

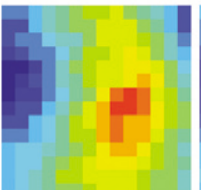

$z=-1 \mu \mathrm{m}$

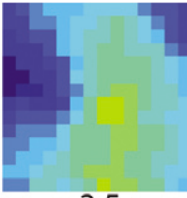

$z=-3.5 \mu \mathrm{m}$

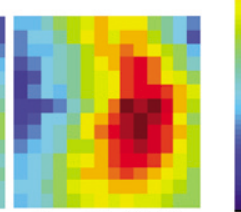

$z=+2.5 \mu \mathrm{m}$
Deformation magnitude

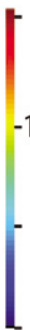

$-1000 \mathrm{~nm}$

$500 \mathrm{~nm}$

Figure 3. Spatial map of the magnitude of the deformation field. Heat maps showing the magnitude of the deformation field at four different $z$ positions within a 50-Pa polyacrylamide gel (here, $z=0$ is defined by the bottom of the magnetic bead, which is embedded within the polymer matrix). For $z>0$, the displacement vectors within the bead volume are interpolated using those in the surrounding gel to maintain continuity of the field. As shown in Figure 2, the bead diameter is $45 \mu \mathrm{m}$, and the 1-nN applied force vector points to the left.

A major challenge in cell traction force measurements is the conversion of the cell-induced deformation field, which is directly measured from microscopy images, to a traction force field, which depends not only on the elastic properties of the hydrogel but also on modeling the distance-dependent decrease in stress away from the source. In general, the substrate is modeled as an infinite half-space subjected to a point surface load and stress fields are calculated only in the plane of the substrate $(22,27)$. Although powerful, this approach has several drawbacks: experimental noise introduced by elastic inhomogeneities or optical aberrations can introduce uncertainties in the calculated traction field (27), thin gels are often employed (28-30), and out-of-plane traction forces-which may play an important regulatory role in cell mechanosensation-are ignored.

To improve our understanding of these important biomaterials, and their use in traction force measurements, we use the portable magnetic tweezers device to apply controlled $\sim \mathrm{nN}$-scale forces directly to the surface of thin polyacrylamide gels using 45- $\mu \mathrm{m}$ magnetic beads that are bonded to the gel surface via biotin-streptavidin linkages (Figure 2A). In this geometry, the bead exerts a force directly on the gel through a micron-scale contact area, mimicking the manner in which cells exert stress at a focal adhesion site (31). We observe the resulting gel deformation by measuring the displacement of small fluorescent latex particles that are embedded within the gel matrix using particle image velocimetry (Figure 2B).

In this geometry, the region directly behind the magnetic particle experienced the largest stretching force (Figure 2C). To determine the shape and extent of the 3-D deformation field, we calculated the 2-D bead displacement as a function of $z$ position, as demonstrated by four representative images in Figure 3. Although the magnitude of deformation is reduced

\section{Force Off}

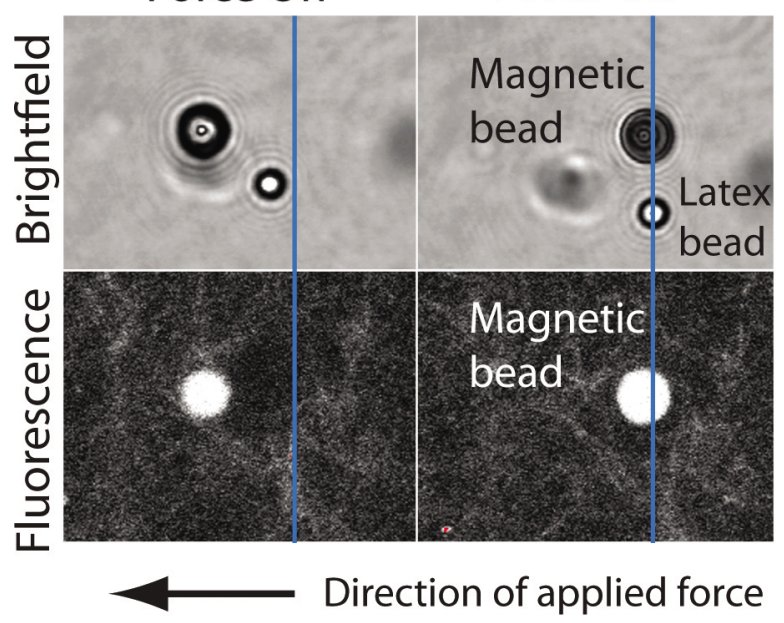

Figure 4. Microscale deformation of an entangled microtubule network. A 4.5- $\mu$ m magnetic bead is embedded in a 3-D network of entangled microtubules ([tubulin] $=15 \mu \mathrm{M}$ ). When a $\sim 10-p \mathrm{~N}$ force is applied to the left, the magnetic bead, which is slightly autofluorescent and can therefore be visualized in both the fluorescence and brightfield channels, moves by a distance roughly equal to its own diameter. At this high strain $(\gamma \approx 1)$, the network in front of the bead is significantly compressed, while a void is created to the rear. A latex particle in close proximity to the magnetic bead is entrained in the deforming microtubule mesh and is also observed to move in the direction of the force (the solid blue line indicates the original position of the latex bead). as a function of slice depth, the strain field propagates over several microns into the gel matrices. In each slice, the general shape of the deformation field is consistent with the expected response of a slab gel to a point force (32): the region behind the adhesive contact zone is substantially stretched and the lateral regions are drawn in toward the center. We see little evidence of compression in front of the bead over the length scales studied here. Future work will focus on understanding the effect of contact area, gel structure and elasticity, and the type/number of adhesive molecules at the bead-gel interface on the 3-D deformation field.

Direct visualization of cytoskeletal deformation under applied load Cellular behavior arises from the coordinated activity of complex protein networks that form structures with a broad range of characteristic length scales, time scales, and interaction energies. The cytoskeleton, a structural biopolymer network that permeates cells, is essential in determining cell shape and strength, enabling intracellular transport of proteins and nutrients, and generating forces during cell division and motility (33). To better understand the molecular origins of the gel stiffness and force sensitivity in this material, we measured the response of networks of microtubules, the stiffest of the cytoskeletal filaments, to localized mechanical perturbations. To achieve this, we generated isotropic and homogeneous entangled gels of fluorescently-labeled microtubules, and seeded these networks with small magnetic and latex beads. Upon application of $\sim 10$-pN forces, we observed the networks to be substantially condensed in front of the particle and rarefied to the rear, suggesting that local compression and bending, rather than tensile restoring forces, may regulate network mechanics at micron-length scales (Figure 4). By directly visualizing microscale network deformation as a function of applied stress, it is possible to identify the molecular origins of elasticity, plasticity, and/or viscous dissipation, as well as the extent of nonaffine deformations. The ability to easily correlate mechanical responses with the local distribution of stress will open new experimental avenues for the study of a wide range of soft materials, including semi-flexible polymer networks, colloidal gels, and living cells.

In summary, this study demonstrates the usefulness of the portable magnetic tweezers device in biological and soft materials characterization by measuring structure-mechanics relationships in synthetic hydrogel films and cellular 
biopolymer networks. Using commercially available magnets and magnetic particles, we applied forces that ranged $\sim 1-1200 \mathrm{pN}$ over approximately micron-scale adhesive areas, typical of the length and force scales found in biomolecular and cellular systems. Because the imaging capabilities of the microscope are unimpaired, this instrument not only allows the measurement of localized stress-strain responses, but enables the correlation of stiffness and forcesensitivity with additional fluorescence readouts (for example, using GFP-labeling to visualize adhesive proteins at the gel interface or crosslinking proteins within a 3-D gel). Finally, the portable tweezers can be constructed at low cost $(<\$ 1000)$ and in many cases, can be used in conjunction with existing microscopy facilities, enabling its use in a broad array of interdisciplinary research and teaching settings.

\section{Acknowledgments}

Authors acknowledge support from the Burroughs Wellcome Fund Career Award at the Scientific Interface (to M.T.V.), the National Science Foundation/National Nanotechnology Infrastructure Network (NSF/NNIN) Research Experiences for Undergraduates (REU) program at University of California-Santa Barbara (grant no. ECS-0335765), a Santa Barbara Cottage Hospital Special Research Award in Biomedical Science, and a National Institutes of Health/National Center for Research Resources (NIH/NCRR) Shared Instrumentation Grant (grant no. 1S10RR017753-01). Authors thank Donna M. Mancusi for assistance in preparing activated glass coverslips, Margaret Gardel for technical advice and useful discussions, and Leslie Wilson and his laboratory for providing tubulin proteins. M.T.V., J.L., and Y.Y. conceived the project; J.L. fabricated the device; all authors prepared samples, collected data, and commented on the manuscript. This paper is subject to the NIH Public Access Policy.

\section{Competing interests}

The authors declare no competing interests.

\section{References}

1. Neuman, K.C. and A. Nagy. 2008. Singlemolecule force spectroscopy: optical tweezers, magnetic tweezers and atomic force microscopy. Nat. Methods 5:491-505.

2. Kollmannsberger, P. and B. Fabry. 2007. Highforce magnetic tweezers with force feedback for biological applications. Rev. Sci. Instrum. 78:114301-114306
3. Trivedi, R.P., T. Lee, K.A. Bertness, and I.I. Smalyukh. 2010. Three dimensional optical manipulation and structural imaging of soft materials by use of laser tweezers and multimodal nonlinear microscopy. Opt. Express 18:27658-27669.

4. Hoffmann, A., G. Meyer zu Hörste, G. Pilarczyk, S. Monajembashi, V. Uhl, and K.O. Greulich. 2000. Optical tweezers for confocal microscopy. Appl. Phys. B 71:747-753.

5. Gullapalli, R.R., T. Tabouillot, R. Mathura, J.H. Dangaria, and P.J. Butler. 2007. Integrated multimodal microscopy, time-resolved fluorescence, and optical-trap rheometry: toward single molecule mechanobiology. J. Biomed. Opt. 12:014012-014017.

6. Conroy, R. 2008. Force spectroscopy with optical and magnetic tweezers, pp. 23-96. In A. Noy (Ed.) Handbook of Molecular Force Spectroscopy. Springer, New York.

7. Bausch, A.R., F. Ziemann, A.A. Boulbitch, K. Jacobson, and E. Sackmann. 1998. Local measurements of viscoelastic parameters of adherent cell surfaces by magnetic bead microrheometry. Biophys. J. 75:2038-2049.

8. Haber, C. and D. Wirtz. 2000. Magnetic tweezers for DNA micromanipulation. Rev. Sci. Instrum. 71:4561.

9. Amblard, F., A.C. Maggs, B. Yurke, A.N. Pargellis, and S. Leibler. 1996. Subdiffusion and anomalous local viscoelasticity in actin networks. Phys. Rev. Lett. 77:4470-4473.

10. Gosse, C. and V. Croquette. 2002. Magnetic tweezers: micromanipulation and force measurement at the molecular level. Biophys. J. 82:3314-3329.

11. Strick, T.R., J.F. Allemand, D. Bensimon, A. Bensimon, and V. Croquette. 1996. The elasticity of a single supercoiled DNA molecule. Science 271:1835-1837.

12. Kim, K. and O. Saleh. 2009. A high-resolution magnetic tweezer for single-molecule measurements. Nucleic Acids Res. 37:E136.

13. Smith, S.B., L. Finzi, and C. Bustamante. 1992. Direct mechanical measurements of the elasticity of single DNA molecules by using magnetic beads. Science 258:1122-1126.

14. Aratyn-Schaus, Y., P.W. Oakes, J. Stricker, S.P. Winter, and M.L. Gardel. 2010. Preparation of complaint matrices for quantifying cellular contraction. J. Vis. Exp. 46:e2173.

15. Boudou, T., J. Ohayon, C. Picart, and P. Tracqui. 2006. An extended relationship for the characterization of Young's modulus and Poisson's ratio of tunable polyacrylamide gels. Biorheology 43:721-728.

16. Reinhart-King, C.A., M. Dembo, and D.A. Hammer. 2005. The dynamics and mechanics of endothelial cell spreading. Biophys. J. 89:676-689.

17. Segur, J.B. and H.E. Oberstar. 1951. Viscosity of glycerol and its aqueous solutions. Ind. Eng. Chem. 43:2117-2120.

18. Drury, J.L. and D.J. Mooney. 2003. Hydrogels for tissue engineering: scaffold design variables and applications. Biomaterials 24:4337-4351.

19. de Chalain, T., J.H. Phillips, and A. Hinek. 1999. Bioengineering of elastic cartilage with aggregated porcine and human auricular chondrocytes and hydrogels containing alginate, collagen, and $\kappa$-elastin. J. Biomed. Mater. Res. 44:280-288.

20. Eaglstein, W.H. and V. Falanga. 1997. Tissue engineering and the development of Apligraf, a human skin equivalent. Clin. Ther. 19:894905.

21. Weinberg, C.B. and E. Bell. 1986. A blood vessel model constructed from collagen and cultured vascular cells. Science 231:397-400.

22. Wang, J.H. and J.S. Lin. 2007. Cell traction force and measurement methods. Biomech. Model. Mechanobiol. 6:361-371.

23. Oliver, T., M. Dembo, and K. Jacobson. 1995. Traction forces in locomoting cells. Cell Motil. Cytoskeleton 31:225-240.

24. Lee, J., M. Leonard, T. Oliver, A. Ishihara, and K. Jacobson. 1994. Traction forces generated by locomoting keratocytes. J. Cell Biol. 127:1957-1964.

25. Oliver, T., K. Jacobson, and M. Dembo. 1998. Design and use of substrata to measure traction forces exerted by cultured cells. Methods Enzymol. 298:497-521.

26. Beningo, K.A. and Y.-L. Wang. 2002. Flexible substrata for the detection of cellular traction forces. Trends Cell Biol. 12:79-84.

27. Sabass, B., M.L. Gardel, C.M. Waterman, and U.S. Schwarz. 2008. High resolution traction force microscopy based on experimental and computational advances. Biophys. J. 94:207220.

28. Buxboim, A., K. Rajagopal, A. Brown, and D. Discher. 2010. How deeply cells feel: methods for thin gels. J. Phys. Condens. Matter 22:194116.

29. Yang, Z., J.-S. Lin, J. Chen, and J.H.C. Wang. 2006. Determining substrate displacement and cell traction fields--a new approach. J. Theor. Biol. 242:607-616.

30. Hur, S.S., Y.Zhao, Y.-S. Li, E. Botvinick, and S. Chien. 2009. Live cells exert 3-dimensional traction forces on their substrata. Cell. Mol. Bioeng. 2:425-436.

31. Stricker, J., B. Sabass, U.S. Schwarz, and M.L. Gardel. 2010. Optimization of traction force microscopy for micron-sized focal adhesions. J. Phys. Condens. Matter 22:194104.

32. Landau, L.D. and E.M. Lifshitz. 1981. Theory of Elasticity. Pergamon, Oxford.

33. Fletcher, D.A. and R.D. Mullins. 2010. Cell mechanics and the cytoskeleton. Nature 463:485-492.

Received 10 March 2011; accepted 16 May 2011

Address correspondence to Megan T. Valentine, Department of Mechanical Engineering Engineering II Building, Room 2361C, University of California-Santa Barbara, Santa Barbara, CA USA.e-mail: valentine@engineering.ucsb.edu.

To purchase reprints of this article, contact: biotechniques@fosterprinting.com 